\title{
Pathologic response after neoadjuvant chemoradiotherapy in Sudanese patients with locally advanced rectal adenocarcinoma
}

\author{
Ahmed Abd Elrahman Abdalla ${ }^{1}$, Awad Ali M. Alawad ${ }^{2 *}$, Hussein Abdalla M. Ali ${ }^{3}$ \\ ${ }^{I}$ Department of Surgery, Faculty of Medicine, University of Gezira \\ ${ }^{2}$ Department of Surgery, Faculty of Medicine, University of Medical Sciences and Technology, Sudan \\ ${ }^{3}$ Department of Surgery, Faculty of Medicine, Sudan International University, Sudan \\ *Corresponding author E-mail:awadali82@hotmail.com
}

\begin{abstract}
Background: Locally advanced rectal cancer can be down staged by neoadjuvant therapy and the resultant tumor response can be quantified histologically.

Objective: This study aimed to assess pathologic response of neoadjuvant chemoradiation in patients with locally advanced rectal cancers treated in Wad Medani Teaching Hospital (WMTH) and National Cancer Institute (NCI), Wad Medani, Sudan.

Patients and Methods: A total of 36 consecutive patients with locally advanced rectal cancer that were managed in WMTH and NCI during the period from 2006-2011 were reviewed. Preoperative pelvic radiotherapy was delivered. The total of 46 Grays were delivered concurrently with 5- fluorouracil (5-FU) on the first and last week of radiation. Total mesorectal excision of the rectal tumour either by anterior or abdominoperineal resections was planned at 6-8 weeks from completion of preoperative treatment. The pathological response to therapy was assessed by histopathology examination of the surgical specimen.

Results: Initial clinical staging of patients revealed $58.3 \%$ of them were stage T3/T4N2M0 and 41.7\% were stage T3N0M0. Downstaging to stage T1/T2N0M0 was found in $36.1 \%$ and stages T3N0M0 in $30.6 \%$. No response was seen in $8.3 \%$ of cases with stage T3/T4N2M0 while a complete clinical response (no residual) was seen in $25.0 \%$. Complete histological response was observed $13.8 \%$. Positive lymph-nodes metastasis was confirmed in $8.3 \%$ of cases.

Conclusion: Neoadjuvant chemoradiation is a reasonable option for cases of rectal cancer and deserves further evaluation.
\end{abstract}

Keywords: Neoadjuvant; Rectal Cancer; Chemoradiation; Pathological Response.

\section{Introduction}

Rectal cancer accounts for nearly 30\% of all colorectal cancer's cases (Elrahman et al. 2012). Surgical resection is the cornerstone of curative therapy (Yoon et al. 2015). Following a potentially curative resection, the 5-year survival rate varies according to disease extent (Yoon et al. 2007). After establishing the diagnosis and completing the staging work-up, a decision is made whether to pursue immediate resection or administer preoperative chemoradiotherapy (CMRT).

The employment of preoperative radiotherapy (RT) combined or not with chemotherapy (CM) has been used in the treatment of rectal cancer for the past two decades and its employ gradually increased as adjuvant therapy, especially in T3/T4 and/or N1/N2 tumors(Suzuki et al. 2014). The strategy of performing preoperative instead of postoperative treatment has the proven advantages of lower acute toxicity, lower total dose of radiation needed and eventual tumor regression and down-staging to enable curative resection and even sphincter preservation.

The objective of this study is to assess the pathological response of neoadjuvant chemoradiation in patients with locally advanced rectal cancers treated in WMTH \& NCI in the period 2006-2011 and to compare our results with the reported standards.

\section{Patients and methods}

This is a retrospective review of patients with locally advanced rectal cancer who were managed in WMTH andlor NCI during the period from 2006-2011. In all 36 patients, records are revised for symptoms. Digital rectal examination findings for all patients were recorded preoperatively at the combined onco-surgical clinic, and all patient's biopsy from the mass was taken as punch biopsy either bed side or during endoscopic examination. Magnetic resonance imaging (MRI) was performed evaluating the stage of the tumor and TNM staging system was used.

Neoadjuvant chemo radiation regimens were as follows: Pelvic radiotherapy delivered with the total central dose of 46 Grays in 23 sessions. Bolus 5-FU was delivered $(400 \mathrm{mg} / \mathrm{m} 2)$ during the first and last weeks of radiation. 5-fluorouracil was given 30 minutes prior to radiation sessions.

All patients had been seen at the combined clinic after 6-8 weeks of radiation. Clinical and radiological assessments were then repeated. Finally, the combined clinic decided on type of surgery according to the initial site of the tumor and response to treatment. Review of the postoperative histopathology report took place addressing the presence of cancer or viable malignant cells, extent of invasion and number of involved lymph nodes; a modified pathologic staging system was used. The Rectal Cancer Regression Grade (RCRG), which simplified the classification to three levels, 
RCRG 1: the tumor is either sterilized or only microscopic foci of adenocarcinoma remain; RCRG 2: marked fibrosis, but with macroscopic tumor still present; and RCRG 3: little or no fibrosis in the presence of abundant macroscopic tumor. RCRG 1 and 2 were considered to represent significant tumor regression (14). Report must include presence of lymph-nodes, and if they were involved or not. Data introduced and analyzed by the computer program (SPSS version 17). To determine the statistical significance of differences, the Pearson test was used and probability test (P. value) with $p<0.05$ considered as significant.

\section{Results}

The total number of cases was thirty six with female to male ratio of $1.25: 1$. All patients were seen in combined clinic. More than $55 \%$ of cases have tumor less than $4 \mathrm{~cm}$ from the anal verge (Figure 1).In this study, $97.2 \%(n=35)$ of patients received full course of CMRT and the dose of radiation ranging between 45-50 Gray Pre and post neoadjuvant therapy clinical staging is shown in (Table 1).

In this study $91.7 \%$ of cases underwent APR, $8.3 \%$ cases underwent Anterior Resection (AR) which was done using staplers, and one patient offered no surgery. Post neoadjuvant therapy histological assessment showed RCRGI in $41.7 \%$ of cases (of them 5 out of 15 , there were complete sterilization of the specimen. 13.8\%), RCRGII in $27.8 \%$, and RCRGIII in $30.6 \%$.
Only $8.3 \%$ were found to have metastatic lymph nodes deposits. In cross tabulation between the results of the histology post CMRT and the grade of the tumors, we found that a significant relationship $(\mathrm{P}=0.031)$ between patient's gradeand response (Table 2).

Table 1:Clinical Staging Using Images Pre and Post CMRT Therapy

\begin{tabular}{|c|c|}
\hline $\begin{array}{l}\text { CMRT therapy Clinical } \\
\text { staging using images } \\
\text { (CT/MRI) } \\
\text { Pre-treatment }\end{array}$ & $\begin{array}{l}\text { Clinical staging using } \\
\text { images (CT/MRI) } \\
\text { post-treatment }\end{array}$ \\
\hline \multirow{4}{*}{15 stage T3NOM0 } & 4 stage TONOMO \\
\hline & 5 stage T1N0M0 \\
\hline & 4 stage $\mathrm{T} 2 \mathrm{~N} 0 \mathrm{M} 0$ \\
\hline & 2 stage T3NOMO \\
\hline \multirow{4}{*}{21 stage T3/T4N2M0 } & 5 stage TONOMO \\
\hline & 4 stage $\mathrm{T} 1 \mathrm{~N} 0 \mathrm{M} 0$ \\
\hline & 9 stage T3NOMO \\
\hline & 3 stage T4N2M0 \\
\hline
\end{tabular}

Table 2:Correlation between Post Neoadjuvant Histological Response and Tumor Grade.

\begin{tabular}{llll}
$\begin{array}{l}\text { Histopathology } \\
\text { post Neoadjuvant } \\
\text { therapy }\end{array}$ & $\begin{array}{l}\text { Tumor grade } \\
\text { Grade 1 }\end{array}$ & Grade 2 & Grade 3 \\
\hline RCRG 1 & 11 & 3 & 1 \\
RCRG 2 & 2 & 6 & 2 \\
RCRG 3 & 2 & 7 & 2 \\
P. value & 0.031 & & \\
\hline
\end{tabular}

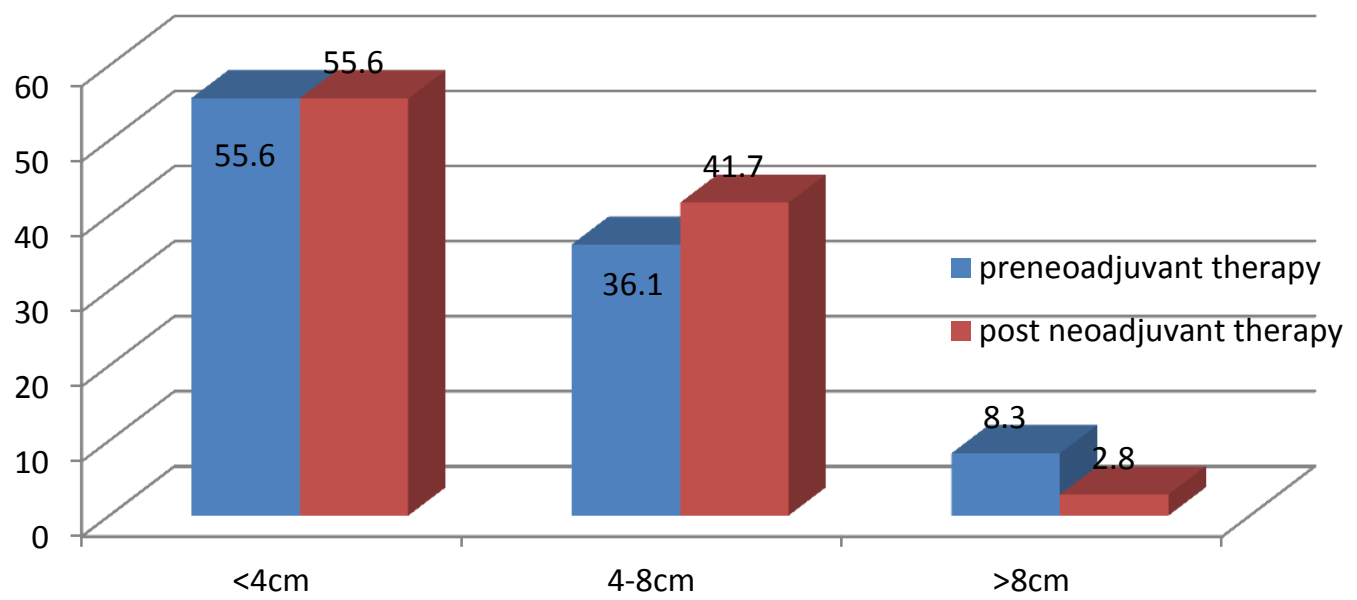

Fig. 1: The Distance from the Anal Verge Pre and Post Neoadjuvant Therapy.

\section{Discussion}

Advances in colorectal cancer treatment create a development of a neoadjuvant CMRT which became widely accepted now. Neoadjuvant CMRT is very effective in reducing the tumor mass as seven out of 36 cases showed no palpable mass, per digital rectal examination (DRE), after neoadjuvant CMRT. All cases were amenable for surgery after neoadjuvant CMRT, including those who presented with fixed tumor $(69.4 \%)$. This reflected the effectiveness of neoadjuvant CMRT in this study.

The results of this study can be compared with Dunst et al study done in Germany, who found clinical response rate of $68 \%(95 \%$ confidence interval: $57-78 \%$ ), and they have used a total irradiation dose of 50.4-55.8 Gy with conventional fractions (Sun et al. 2014). Capecitabine was given at an oral dosage of $825 \mathrm{mg} / \mathrm{m}^{2}$ bid on each day of the radiotherapy period with the first daily dose applied $2 \mathrm{~h}$ before irradiation, followed by surgery six weeks later (Maretto et al. 2007).

In this series $58.3 \%$ of patients $(n=21)$ were found to have stageT3/T4N2M0 and post therapy, only 3 cases had this stage $8.3 \%$ and in $41.7 \%$ of patients $(n=15)$ with stage T3NOM0 prior to treatment, the down-staging was seen in (13/15). Radiological complete resolution was observed in $25.0 \%$ of cases. The overall down-staging in this study was observed in (31/36). In comparison with a study done by Garland et al, they showed down-staging was found in $56.7 \%$ of cases (Garland et al. 2014). Duke's university study showed down-staging in $82 \%$ of cases, and this was compatible with our findings (Ciccocioppo et al. 2009).

In study conducted in Shanghai, they studied 105 patients, of these 13 patients showed a complete tumor response after neoadjuvant therapy, and they spared the operation (Choi et al. 2012). In our study, we were following the case which experienced complete clinical and pathological response, and who remained free since 2009. Pathological complete response which was observed in this study was comparable to the findings of Dunst et al they have the pathologically complete response was achieved in six patients (7\%, 95\% confidence interval: 3-14\%) (Bujko et al. 2007).

In cross tabulation between the results of the histology post CMRT and the grade of the tumors, we found a significant relationship $(\mathrm{P}=0.031)$ between patient's grade and response. Eleven out of 15 tumors with grade I showed RCRGI, on the other hand, only 3 out of 16 tumors grade 2 showed RCRGII, while only one tumor with grade 3 (out of five) showed complete response RCRGIII. This signifies that the tumor grade may predict the response to treatment (Table 2). 
In $8.3 \%$ of our patients there were lymph nodes retrieved in the specimen after surgical resection, this correlate well with the series reported by De la Fuente SG et al, who found fewer total lymph-nodes were retrieved in the neoadjuvant therapy patients compared to those who did not receive preoperatively therapy (Neo 14.6 +/- 0.6 vs. No-Neo 17.2 +/- 1.1) (Chao et al. 2014).

\section{Conclusion}

Neoadjuvant chemoradiation in locally advanced rectal cancer in Sudanese patients provide a significant pathologicl response, and it deserves further evaluation.

\section{References}

[1] Elrahman AAEAA, Alshaihk AA, Abuidris D \& Elfatih M (2012) Neoadjuvant chemoradiation for rectal cancer: analysis of clinical outcomes for patients treated in Wad Medani Teaching Hospital and National Cancer Institute (Sudan) in the period 2006-2011. Sudan Medical Journal 48, 129-134.

[2] Bujko K, Michalski W, Kepka L, Nowacki MP, NasierowskaGuttmejer A, Tokar P, Dymecki D, Pawlak M, Lesniak T, Richter P, Wojnar A, Chmielik E \& Polish Colorectal Study G (2007): Association between pathologic response in metastatic lymph nodes after preoperative chemoradiotherapy and risk of distant metastases in rectal cancer: An analysis of outcomes in a randomized trial. Int $J$ Radiat Oncol Biol Phys 67, 369. 377.http://dx.doi.org/10.1016/j.ijrobp.2006.08.065.

[3] Chao JY, Wang HM, Chiang FF, Lin JC, Chang CF, Lin JF \& Yeh HL (2014): Preoperative chemoradiotherapy with oxaliplatin and tegafur-uracil in locally advanced rectal cancer: pathologic complete response rate and preliminary results of overall and disease-free survival in a single institute in Taiwan. J Chin Med Assoc 77, 128132.http://dx.doi.org/10.1016/j.jcma.2013.11.008.

[4] Choi CH, Kim WD, Lee SJ \& Park WY (2012): Clinical predictive factors of pathologic tumor response after preoperative chemoradiotherapy in rectal cancer. Radiat Oncol $J$ 30, 99107.http://dx.doi.org/10.3857/roj.2012.30.3.99.

[5] Ciccocioppo A, Stephens JH, Hewett PJ \& Rieger NA (2009) Complete pathologic response after preoperative rectal cancer

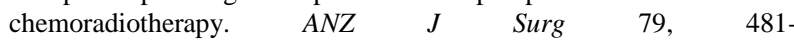
484.http://dx.doi.org/10.1111/j.1445-2197.2009.04950.x.

[6] Garland ML, Vather R, Bunkley N, Pearse M \& Bissett IP (2014) Clinical tumour size and nodal status predict pathologic complete response following neoadjuvant chemoradiotherapy for rectal cancer. Int J Colorectal Dis 29, 301-307.http://dx.doi.org/10.1007/s00384013-1821-7.

[7] Maretto I, Pomerri F, Pucciarelli S, Mescoli C, Belluco E, Burzi S, Rugge M, Muzzio PC \& Nitti D (2007): The potential of restaging in the prediction of pathologic response after preoperative chemoradiotherapy for rectal cancer. Ann Surg Oncol 14, 455461.http://dx.doi.org/10.1245/s10434-006-9269-4.

[8] Sun Y, Chi P, Xu B, Lin H, Lu X, Huang Y, Xu Z, Huang S \& Jiang $C$ (2014): [Predictive factors associated with pathologic complete response after neoadjuvant chemoradiotherapy in rectal cancer]. Zhonghua Wei Chang Wai Ke Za Zhi 17, 556-560.

[9] Suzuki T, Sadahiro S, Tanaka A, Okada K, Saito G, Kamijo A, Akiba T \& Kawada S (2014): Relationship between histologic response and the degree of tumor shrinkage after chemoradiotherapy in patients with locally advanced rectal cancer. J Surg Oncol 109, 659664.http://dx.doi.org/10.1002/jso.23550.

[10]Yoon SM, Kim DY, Kim TH, Jung KH, Chang HJ, Koom WS, Lim SB, Choi HS, Jeong SY \& Park JG (2007): Clinical parameters predicting pathologic tumor response after preoperative chemoradiotherapy for rectal cancer. Int J Radiat Oncol Biol Phys 69, 1167-1172.http://dx.doi.org/10.1016/j.ijrobp.2007.04.047.

[11] Yoon WH, Kim HJ, Kim CH, Joo JK, Kim YJ \& Kim HR (2015): Oncologic impact of pathologic response on clinical outcome after preoperative chemoradiotherapy in locally advanced rectal cancer. Ann Surg Treat Res 88, 1520.http://dx.doi.org/10.4174/astr.2015.88.1.15. 